\title{
A Lucratividade Inerente e Implícita no Estoque na Análise de Liquidez Estática
}

\author{
Adail Marcos Lima da Silva \\ Guilherme deAlbuquerque Cavalcanti
}

\section{Resumo}

O objetivo central deste trabalho é equacionar a perspectiva estática em um processo de aprimoramento da manipulação do estoque na tradicional análise de liquidez. Especificamente, a margem de lucratividade do estoque é utilizada para corrigir os saldos dos estoques, apregoando a substituição estática pela dinâmica. O artigo apresenta referencial teórico sobre análise de liquidez e solvência e a relação da lucratividade com esses fatores. O estudo é teórico e conduz a inserção da lucratividade em cálculos tradicionais de capital circulante líquido, liquidez geral, liquidez corrente e liquidez de Kanitz, que matematicamente modificados se adaptam à lógica dinâmica, o que permite o cálculo de valores mais confiáveis à mensuração da solvência de curto prazo.

Palavras-chave: liquidez estática; estoque; lucratividade inerente e implícita; otimização dinâmica.

\begin{abstract}
The mainly target of this work is to solve the static perspective on a process to optimize the inventory manipulation at the traditional liquidity analysis. Specifically, the inventory liquidity margin has been used to correct stock on hand, announcing the switch of static for dynamic way. This article presents theoretical reference about solvency and liquidity analysis and which relation the profitability has with these factors. This research is theoretical and guides a profitability insertion on traditional calculations of net work capital, general liquidity, immediately liquidity and Kanitz liquidity, that if was mathematically changed would be adapt to the dynamic's logic, what would allow the calculation of more reliable values to the short time limit's solvency measurement.
\end{abstract}

Key words: static liquidity; inventory; implicit and inherit profitability; dynamic optimization. 


\section{INTRODUÇÃO}

Em 1908, Willian Rosendale veicula um artigo na revista Banker's Magazine, publicada pela associação dos Banqueiros de Nova York, intitulado Credit Department Methods, almejando orientar os intermediários do mercado financeiro na consecução de análises destinadas à capacidade de pagamento - solvência das empresas, mediante solicitações de crédito (Myers, 1974). Foram as primeiras alusões mercadológicas despendidas na análise das demonstrações contábeis, cujos ativos e passivos circulantes foram manipulados conjuntamente sob a forma de quociente.

A divisão do ativo circulante pelo passivo circulante popularizou o mais antigo dos indicadores oriundos do processamento de comparações lógicas entre rubricas específicas do balanço patrimonial, notoriamente conhecido por liquidez corrente (Myers, 1974). Outros medidores, baseados no poder de cobertura dos compromissos, foram confeccionados e integram o conjunto dos tradicionais medidores da análise de liquidez. Empregados no estudo da solvência - capacidade de pagamento dos compromissos assumidos em face da estrutura de financiamento montada - os índices de liquidez foram constituídos para responder à seguinte indagação: se uma empresa encerrar suas atividades, deterá capacidade de superação das dívidas?

O caráter estático, associado aos medidores de liquidez, em função da falência, pode ser evidenciado a partir do valor dos estoques. Se estes são cogitados nos cálculos com valores atrelados ao custo dos produtos vendidos, a lucratividade sobre as vendas é desprezada; portanto não há consideração da consecução das transações comerciais por vias normais. Em caso de alteração no foco da análise, ou seja, caso a solvência seja estimada sob a hipótese da prosperidade empresarial - perspectiva dinâmica - os índices convencionais de liquidez expressarão valores situados à margem da realidade.

Para amoldá-los à lógica dinâmica, os medidores de liquidez devem trabalhar com os estoques devidamente corrigidos, onde a lucratividade inerente precisa deixar de ser implícita.

Com o emprego da margem de lucratividade do estoque calculada com a divisão do lucro antes das despesas, dos juros e do imposto de renda pelo custo dos produtos vendidos, os medidores de liquidez se adaptam à dinâmica, e passam a tratar o lucro bruto como meio de superação dos compromissos emanados da porção externa de recursos usados na composição da estrutura de capital. 
Como o capital circulante líquido, a liquidez geral, a liquidez corrente e a liquidez de Kanitz processam o volume dos estoques em suas formulações matemáticas, permitem a concretização da adaptação. Sendo assim, quatro medidores tradicionalmente estáticos serão adaptados à dinâmica empresarial.

\section{AnÁlise de Liquidez e Solvência}

O estudo da solvência procura identificar se uma empresa se encontra apta à consecução das liquidações emanadas das fontes de financiamento, quando pressionada pelo encerramento dos vencimentos específicos (Silva, 2002).

Por meio do balanço patrimonial, a solvência encontra estimação a partir de comparações entre realizações e liquidações, respectivamente ativas e passivas. Observando as devidas exceções, todo o ativo será realizado por meio da ocorrência de entradas de caixa; todo o passivo será liquidado por meio da ocorrência de saídas de caixa. Os agrupamentos de ordem corrente encontram-se mais suscetíveis às consecuções de realizações e de liquidações; contrariamente, ativos e passivos não-correntes exprimem menores possibilidades; portanto ativos e passivos possuem graus de realização e de liquidação.

Por se preocupar com a capacidade financeira na cobertura das obrigações assumidas ante as fontes de recursos, a solvência encontra avaliação na comparação entre os graus de realização e de liquidação. Do lado do ativo, quanto maior o grau de realização - maior volume de recursos situados na porção circulante - melhor será o conceito de solvência. Do lado do passivo, quanto menor o grau de liquidação - menor volume de captações correntes - melhor será o conceito de solvência. Finalmente, na solvência arranjos patrimoniais, exprimindo altos e baixos graus de realização e de liquidação, constituem as melhores configurações de situação financeira de uma empresa.

Tanto o grau de realização como o de liquidação encontram-se dependentes de duas características: da agilidade com que imputarão alterações no saldo do caixa e da capacidade de dar manutenção aos valores previstos. Tais propriedades derivam das dimensões da liquidez apregoadas por Van Horne (1979, p. 521) e Weston e Brigham (2000, p. 51), cuja discussão se concentra no esclarecimento da capacidade de converter ativos não-monetários em CX o mais rápido possível - agilidade - por um justo preço de mercado - manutenção de valores - perfazendo o conceito de liquidez contábil inerente aos ativos (Ross; Westerfield; Jaffe, 2002, p. 39).

A agilidade reporta-se aos prazos médios indicativos dos intervalos necessários às consecuções das entradas de caixa, por parte dos ativos não-monetários, e 
das saídas de caixa, por parte dos passivos. Destarte, o arranjo favorável à solvência tem constituição com base na interação de prazos médios menores para ativos não-monetários e maiores para passivos (Matarazzo, 1998).

A capacidade de dar manutenção à consumação dos valores nominais previstos para ativos não-monetários e passivos, vincula-se à apuração conclusiva da solvência. Financeiramente, toda empresa se interessa em receber de forma integral as cifras ativas não-monetárias - capacidade de manutenção eficiente e pagar com a maior barganha possível os compromissos com o passivo capacidade de manutenção deficiente. Logo, na solvência o arranjo ideal dá-se pelas configurações eficiente e deficiente das capacidades de manutenção ativas e passivas, respectivamente (Brigham; Hoston, 1999).

Para extrair informações aproximadas na efetiva condição de solvência com base em demonstrações contábeis, duas análises mostram-se imprescindíveis: análise de eficiência operacional ou de atividade e análise de liquidez. Sustentar posições de solvência a partir do emprego isolado das duas formas analíticas pode não ser a melhor alternativa. Utilizando somente a agilidade - análise de eficiência operacional ou de atividade - serão observadas noções unicamente relacionadas com as ocorrências dos recebimentos e dos pagamentos, isto é, fluxo de caixa. Com a capacidade de manutenção - análise de liquidez - tem-se isoladamente evidenciada a proporção da quantidade de direitos na cobertura das obrigações, isto é, relações entre os fluxos. Finalmente, quando uma circunstância almeja conceber proposições atinentes à condição da solvibilidade empresarial, as duas vertentes analíticas devem prover de modo mútuo as informações necessárias.

Em função de comparar graus de realização e de liquidação, os convencionais medidores de liquidez são empregados no estudo da solvência, sob a perspectiva da capacidade de manutenção de valores ativos e passivos. Ao todo, são seis indicadores: capital circulante líquido (CCL); liquidez geral (LG); liquidez corrente (LC); liquidez seca (LS); liquidez de Kanitz (LK); e liquidez absoluta (LA). O CCL expressa valores absolutos monetários, enquanto os demais medidores exprimem frações.

\section{Capital Circulante Líquido}

Os dispêndios de ordem corrente representam todos os investimentos de curto prazo e se encontram contabilmente alocados no balanço patrimonial sob o título de ativo circulante - AC. Podem ser financiados com recursos captados junto a fontes tanto de curto como de longo prazo (Gitman, 1997).

Quando fontes de longo prazo participam da estrutura de captações formatada unicamente ao sustento do AC, ocorre a combinação de direitos realizáveis cíclicos 
com liquidações não-cíclicas; trata-se de situação favorável à solvência (Fleuriet; Brasil, 1993). Destarte, maiores níveis de fontes não-cíclicas presentes no AC promovem uma classificação de solvibilidade otimizada; contrariamente, pouca ou nenhuma fonte não-cíclica no AC inspira preocupações mediante a conceituação negativa assumida pela solvência.

Financeiramente, o passivo circulante - PC - deve ser tratado como fonte de fundos exclusiva do AC. Assim, caso o AC supere o PC, existirão recursos captados junto a fontes de longo prazo; em condição de igualdade ou inferioridade, fontes não-cíclicas encontrar-se-ão investidas somente no ativo não-cíclico (Fleuriet; Kehdy; Blanc; 2003).

Para saber se há ou não fonte de longo prazo alocada no AC, basta diminuí-lo pelo saldo do PC, dando origem ao capital circulante líquido - CCL, fórmulas 01 e 02, onde: CX, caixa; QC, quase-caixa; DR, duplicatas a receber; EST, estoque; ORC, outros recebíveis correntes; EFC, empréstimos e financiamentos circulantes; FOR, fornecedor; PRO, provisões em geral; OLC, outros liquidáveis correntes. O conjunto formado por CX, QC, DR, EST e OLC representa uma composição básica do AC; EFC, FOR, PRO e OLC integram uma versão igualmente básica do PC.

$C C L=A C-P C$

$C C L=C X+Q C+D R+E S T+O R C-E F C-F O R-P R O-O L C$

O CCL representa justamente o volume de recursos destinados ao AC pelas fontes de longo prazo. Os dispêndios de capital ou ativos não-cíclicos possuem a preferência na distribuição dos recursos de longo prazo às opções de investimento. Logo, o CCL pode ser igualmente calculado a partir da diferença entre os valores não-cíclicos passivos e ativos - fórmula 03.

$C C L=E L P+P L-R L P-A P$

Sob a perspectiva da fórmula 09, é possível enumerar duas alternativas para o arranjo do CCL (Silva, 2003): unilateral e bilateral. Sendo unilateral, uma única opção de fonte não-cíclica - ELP ou PL - estará compondo o CCL. Bilateralmente, tanto o ELP como o PL, em proporções não necessariamente similares, farão parte do CCL.

Quanto maior a porção do AC financiada com CCL, melhor será a solvência. Quanto menor a porção do AC financiada com CCL, pior será o conceito de solvibilidade. 


\section{Liquidez Geral}

O índice de liquidez geral - LG - mensura a representatividade da soma entre AC e RLP em face do valor montado pelo passivo exigível - PC e ELP. Por relacionar rubricas tanto de longo como de curto prazo, a LG exprime uma noção global de solvência (Walter; Braga, 1979).

Considerando as devidas exceções à regra, ativos e passivos são valores nominais respectivamente promotores de entradas e saídas de caixa, com datas e volumes específicos; AC e PC são entradas e saídas nominais de ordem corrente; assim, evidenciam uma relação ligada com a solvência no curto prazo; RLP e ELP são entradas e saídas nominais de caráter não-corrente, sugerindo uma relação vinculada com a solvência no longo prazo. Assim, por tentar apregoar de modo quantitativo a solvência simultaneamente de curto e de longo prazo, à LG podem ser imputadas as seguintes classificações: solvência geral e índice de solvibilidade ampla.

As duas mais práticas perspectivas matemáticas da LG estão representadas nas fórmulas 04 e 05 .

$$
\begin{aligned}
& L G=\frac{A C+R L P}{P C+E L P} \\
& L G=\frac{C X+Q C+D R+E S T+O R C+R L P}{E F C+F O R+P R O+O L C+E L P}
\end{aligned}
$$

A fórmula 06, além de ser uma terceira modalidade de cálculo da LG, também revela uma interpretação importante.

$$
L G=\frac{P C+C C L+R L P}{P C+E L P}
$$

Por ser o AC a soma entre o PC e o CCL, a participação do CCL na fórmula 06 suscita a descrição das duas modalidades pertinentes à formação dele. Unilateralmente, apenas um tipo de fonte não-cíclica poderá participar. Bilateralmente, sem nenhuma observação proporcional preliminar, as duas opções de fontes não-cíclicas podem ser praticadas. No encerramento de uma dada variação temporal: o AC apresentar variação positiva sustentada pelo CCL; com o PL sendo o responsável pelo incremento do CCL; com o PC, o ELP e o RLP sem alterações significativas, a LG exprimirá valor maior. Ao substituir o CCL na fórmula 06 por sua fórmula 03, tem-se um melhor entendimento da relação entre LG e PL - fórmulas 07, 08 e 09. 
$L G=\frac{P C+P L+E L P-R L P-A P+R L P}{P C+E L P}$

$L G=\frac{P C+P L+E L P-A P}{P C+E L P}$

$L G=1+\frac{P L-A P}{P C+E L P}$

Destarte, reforços ao AC com PL contribuem com a LG: caso algum novo aporte seja unilateralmente concretizado com o PL ou, se bilateralmente, o PL assume maior proporção em face ELP, a LG será sempre otimizada, mesmo sem ter aumentado o valor, pois o interesse reside somente na forma como o CCL tem constituído seu volume e nas conseqüentes implicações impostas à solvência geral. Concluindo, quanto maior a superioridade do PL em face do AP, maior será a LG.

\section{Liquidez Corrente}

Com o objetivo de averiguar a capacidade de cobertura dos compromissos assumidos no curto prazo, o índice de liquidez corrente - LC - relaciona as contas principais da estrutura patrimonial de curto prazo (Silva, 1999). Dividindo o AC pelo PC, extrai-se uma noção do poder de superação das exigibilidades correntes. Quanto maior a LC, melhor será à solvência no curto prazo; quanto menor a LC, pior será à solvência no curto prazo.

As fórmulas 10 e 11 mostram as duas modalidades matemáticas atreladas à interpretação principal da LC.

$$
\begin{aligned}
& L C=\frac{A C}{P C} \\
& L C=\frac{C X+Q C+D R+E S T+O R C}{E F C+F O R+P R O+O L C}
\end{aligned}
$$

Na fórmula 12, tem-se uma abordagem matemática oriunda de conceituação diferente.

$L C=\frac{P C+C C L}{P C}$

A partir do raciocínio ligado ao arranjo do AC dado pela soma entre PC e CCL, a LC destina-se à quantificação da superioridade - se houver - dos recursos captados unicamente na composição do AC, sobre a parcela de curto prazo 
manipulada; adota-se a hipótese da total cobertura do PC com os recursos do próprio, pois os fundos levantados junto às fontes correntes são investidos unicamente no AC. Assim, uma LC superior a 1 tem ocorrência garantida, se e somente se o CCL for superior a zero (Matarazzo, 1998); a LC será igual a 1, se e somente se o CCL for igual a zero (Matarazzo, 1998); a LC será inferior a 1, se e somente se o CCL for inferior a zero (Matarazzo, 1998), estabelecendo uma relação a descoberto entre AC e PC (Walter; Braga, 1979).

As três possibilidades fundadas em torno da LC, a partir das magnitudes assumidas pelo CCL, tem melhor visualização na fórmula 13.

$$
L C=1+\frac{C C L}{P C}
$$

\section{Liquidez Seca}

O índice de liquidez seca - LS - exclui do AT o saldo do EST, para depois dividir o resultado pelo PC, fórmula 14. Com base no fato de ser a rubrica com menor grau de realização na obrigação de liquidar os compromissos, o EST tem seu valor retirado no cálculo da LS, pois o medidor almeja estimar um potencial de pagamento mais ágil - DR pode ser mais facilmente convertida em CX, o CX e o QC são as representações da liquidez em seu estado pleno - das fontes correntes (Silva, 2002).

$L S=\frac{A C-E S T}{P C}$

Baseando-se somente no episódio da falência, a LS prega um raciocínio interpretativo, cuja preocupação central reside na mensuração do poder de cobertura das obrigações de curto prazo, sob uma configuração inerte; destarte, a exclusão do EST tem justificativa centrada na hipótese da não consecução de vendas; a liquidação do PC será concretizada mediante o emprego dos saldos manifestados pelo CX, QC e DR - fórmula 15, disjuntos em face das vendas.

$L S=\frac{C X+Q C+D R+O R C}{E F C+F O R+P R O+O L C}$

Reformulando o cálculo da LS, a partir da estrutura de financiamento montada ao AC, o saldo da conta EST será retirado da soma entre PC e CCL, fórmula 16.

$L S=\frac{P C+C C L-E S T}{P C}$ 
Adotando o ponto de vista da fórmula 16, observa-se uma total dependência da LS perante o CCL: se o CCL for inferior ao EST, a LS será inferior a 1; se o CCL for igual ao EST, a LS será igual a 1; se o CCL for superior ao EST, a LS será superior a 1. A afinidade entre LS e CCL tem demonstração matemática precisa na fórmula 17.

$L S=1+\frac{C C L-E S T}{P C}$

Por ser salutar a manutenção da LS próxima, igual ou superior a 1 , solidificando uma formatação adequada à solvência de curto prazo, a utilização de fontes de financiamento com liquidação de longo prazo faz-se indispensável no arranjo do giro, ou na composição do AC.

\section{Liquidez de Kanitz}

Na tentativa de esboçar um medidor dinâmico de liquidez, a partir da desconsideração da estática associada à falência, o índice de liquidez de Kanitz - LK - aborda os medidores LC e LS sob a égide da prosperidade empresarial, identificando equívocos e sugerindo uma mensuração intermediária de solvência.

Por intermédio da adoção de duas visões antagônicas despendidas para os índices LC e LS, a LK pode ser decifrada como indicador capaz de fundir as duas visões, sendo ao mesmo tempo LC e LS.

Em ambiente empresarial próspero, a LC dedica ao EST uma expectativa otimista, ao considerar uma consecução integral de vendas. De modo oposto, a LS não prevê a venda de nem mesmo uma única unidade do EST, expressando expectativa pessimista.

Ambas as notações podem perfeitamente ser enquadradas como equívocos. A ótica otimista tem sua premissa indeferida, em função da possibilidade de o EST não ser totalmente vendido por motivos mercadológicos, contração da demanda, ou operacionais, como perdas oriundas da característica perecível inerente a certos tipos de produtos. Por exemplo, vislumbrar a conversão integral em CX - venda a vista - e em DR - venda a prazo - do saldo da conta EST, implica uma característica desfavorável a LC. A ótica pessimista descarta quaisquer estimativas favoráveis à realização de vendas do EST. Em seu contexto operacional, o EST é vendido e transformado em CX ou em DR, posteriormente convertidas em CX, tornando sem sentido a exclusão total no cálculo da LS; definitivamente, a LS tem sua funcionalidade desvirtuada, ao descartar os recursos arrecadados com as vendas para a manutenção da solvência. 
Com o objetivo de prover uma atitude intermediária na análise da solvência, sugerindo uma perspectiva posicionada entre as vertentes otimista e pessimista, a LK exclui apenas uma porção do EST com a venda racionalmente tratada como impraticável. Somente uma fração, estimada na concretização de vendas do saldo da conta EST - F , nas fórmulas 18, 19, 20 e 21 - participará do estudo da solvência.

Na fórmula 18, o AC tem seu valor reduzido por meio da exclusão do volume do EST não vendido (1 menos $\mathrm{F}_{\mathrm{V}}$ ).

$L K=\frac{A C-E S T \times\left(1-F_{V}\right)}{P C}$

Na fórmula 19, somente o volume vendido do EST (EST vezes $\mathrm{F}_{\mathrm{v}}$ ) encontra-se somado com CX, QX, DR e ORC.

$L K=\frac{C X+Q C+D R+O R C+E S T \times F_{V}}{E F C+F O R+P R O+O L C}$

As fórmulas 20 e 21 esboçam a LK, considerando no numerador as fontes de financiamento do AC. Na fórmula 20, da soma entre PC e CCL é retirada a porção não-vendida do EST.

$L K=\frac{P C+C C L-E S T \times\left(1-F_{V}\right)}{P C}$

A fórmula 21 deriva da 20 e apregoa as seguintes conjecturas: se o CCL for inferior a porção não-vendida do EST, a LK será inferior a 1. Se o CCL for igual à porção não-vendida do EST, a LK será igual a 1 . Se o CCL for superior à porção não-vendida do EST, a LK será superior a 1.

$L K=1+\frac{C C L-E S T \times\left(1-F_{V}\right)}{P C}$

Podendo ser considerada uma tentativa de enquadrar a dinâmica na análise da solvência por meio dos medidores convencionais de liquidez, a LK comporta-se como variável dependente, quando relacionada com o CCL. Quanto maior o CCL, maior será a LK e melhor será a solvência.

\section{Liquidez Absoluta}

Também conhecida como liquidez imediata e instantânea, a liquidez absoluta LA - divide a soma entre CX e QC pelo PC (fórmula 22), visando a exprimir a 
capacidade de honrar as liquidações correntes de maneira momentânea (Walter; Braga, 1979), no alcance das datas dos vencimentos específicos - EFC, FOR e PRO.

$$
L A=\frac{C X+Q C}{E F C+F O R+P R O+O L C}
$$

Do total manifestado pelo AC são retirados os saldos das contas DR e EST, para posterior divisão com o PC. Trata-se de uma segunda perspectiva de cálculo da LA, apresentada pela fórmula 23.

$L A=\frac{A C-D R-E S T}{P C}$

Empregando as possíveis fontes de financiamento do AC para expressar a LS, tem-se excluído da soma entre o PC e o CCL a soma entre DR e EST - fórmula 24.

$L A=\frac{P C+C C L-D R-E S T}{P C}$

A fórmula 25 deriva da 24 e sugere as seguintes ponderações: a LS será inferior a 1, se o CCL for inferior a soma entre DR e EST; a LS será igual a 1, se o CCL for igual à soma entre DE e EST; a LS será superior a 1, se o CCL for superior a soma entre DR e EST.

$L A=1+\frac{C C L-D R-E S T}{P C}$

A LA tem seu valor dependente do CCL. Assim, quanto maior a participação do CCL no fomento do AC, maior será a possibilidade de a LA situar-se em patamares favoráveis à solvência.

\section{Lucratividade Versus Análise de Liquidez Versus Solvência}

Os convencionais medidores de liquidez manipulam rubricas patrimoniais e são empregados na apreciação aproximada da solvência. Basicamente, os cálculos deles destinam-se à divulgação do seguinte raciocínio: caso uma empresa encerre suas atividades em face da incorporação de uma configuração estrutural congruente com o conceito de falência, poderá cobrir as obrigações emanadas da estrutura de capital montada? Por ser cogitada, a falência imputa aos tradicionais índices de liquidez uma denotação estática. 
Para averiguar o poder de cobertura dos compromissos correntes, os tradicionais medidores de liquidez são corriqueiramente empregados. Tipicamente, descartam a participação da lucratividade implícita nos estoques como meio de liquidações. Quando sob a égide da condição estática, a desconsideração da lucratividade encontra justificativa; todavia, em sintonia com a dinâmica, a análise de liquidez convencional apresenta conclusões de solvência situadas à margem da realidade.

No livro Estudo Analítico da Rentabilidade das Empresas, Lopes de Sá (1971) faz referências à lucratividade implícita associada ao volume do EST, classificando-a como disponibilidades ocultas no decurso das atividades operacionais.

Ao ser cogitado o termo decurso, sugere ele uma configuração dinâmica na análise de liquidez. Destarte, na dinâmica da solvência, a lucratividade mostra-se indispensável à formulação matemática da lógica emanada da hipótese de prosperidade empresarial.

Sendo o custo dos produtos vendidos - CPV - a representação do EST na demonstração do resultado do exercício, o lucro antes das despesas, dos juros e do imposto de renda - LADJIR (ou lucro bruto) - assume a condição de lucro inerente e implícito no volume da conta EST (Silva, 2003). Logo, a margem de lucratividade do EST - $\mathrm{ML}_{\mathrm{EST}}$, órmula 26 - será igual ao LADJIR dividido pelo CPV (Silva, 2003).

$M L_{E S T}=\frac{L A D J I R}{C P V}$

Durante determinado período de tempo, uma situação de liquidez pode ser alterada significativamente, se os lucros se inserirem nos cálculos dos convencionais medidores de liquidez (Lopes de Sá, 1971). Quando matematicamente processados, os indicadores de liquidez desprezam a lucratividade implícita no EST, justificando o conceito de disponibilidade oculta. Assim, existem dois conjuntos de índices de liquidez destinados ao estudo da solvência: estáticos e dinâmicos. Os estáticos promovem as disponibilidades ocultas e vinculam-se à configuração da falência (Brasil; Brasil, 1992). Os dinâmicos inserem a lucratividade na apuração dos medidores e encontram-se atrelados à prosperidade empresarial.

Com o intuito de adaptar os medidores tradicionais de liquidez estática à formatação dinâmica, a $\mathrm{ML}_{\mathrm{EST}}$ vai inserir-se na correção do EST. Nos índices de liquidez dinâmica, inserir-se-á a lucratividade do EST - $\mathrm{L}_{\mathrm{EST}}$ - e o EST corrigido

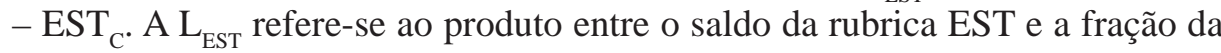
$\mathrm{ML}_{\mathrm{EST}}$ - fórmula 27. O EST $\mathrm{C}_{\mathrm{C}}$ resulta da soma entre $\mathrm{EST}$ e $\mathrm{L}_{\mathrm{EST}}$ - fórmula 28. As 
disponibilidades antes ocultas farão parte do estudo da solvência, sendo uma proposição elucidativa à deficiência estática.

$$
\begin{aligned}
& L_{E S T}=E S T \times M L_{E S T}=E S T \times \frac{L A D J I R}{C P V} \\
& E S T_{C}=E S T \times\left(1+M L_{E S T}\right)=E S T \times\left(1+\frac{L A D J I R}{C P V}\right)
\end{aligned}
$$

O CCL, a LG, a LC e a LK integram o conjunto dos indicadores estáticos e serão tratados por medidores de liquidez amoldados à lucratividade. Depois de adaptados, os resultados apurados pelos medidores serão superiores aos estáticos, pois a mudança central reporta-se à inclusão da rentabilidade do EST nos numeradores das fórmulas de liquidez estática.

\section{Capital Circulante Líquido Adaptado}

No capital circulante líquido adaptado, $\mathrm{CCL}_{\mathrm{A}}$, além das fontes de longo prazo aplicadas no AC, a lucratividade bruta sobre as vendas marcará presença, incorporando a função de alternativa na cobertura das obrigações correntes.

Matematicamente apresentado pelas fórmulas 29 a 34, o CCL $\mathrm{C}_{\mathrm{A}}$ exprimirá situação favorável à solvência, quando situado em níveis considerados elevados; assim, quanto maior melhor.

$$
\begin{aligned}
& C C L_{A}=A C+L_{E S T}-P C \\
& C C L_{A}=A C+E S T \times M L_{E S T}-P C \\
& C C L_{A}=A C+E S T \times \frac{L A D J I R}{C P V}-P C \\
& C C L_{A}=C+Q C+D R+E S T_{C}+O R C-E F C-F O R-P R O-O L C \\
& C C L_{A}=C X+Q C+D R+E S T \times\left(1+M L_{E S T}\right)+O R C-E F C-F O R-P R O-O L C \\
& C C L_{A}=C X+Q C+D R+E S T \times\left(1+\frac{L A D J I R}{C P V}\right)+O R C-E F C-F O R-P R O-O L C
\end{aligned}
$$

$\mathrm{O} \mathrm{CCL}_{\mathrm{A}}$ será sempre superior ao CCL. Para calcular o valor incremental, advindo da lucratividade do EST, basta diminuir o $\mathrm{CCL}_{\mathrm{A}}$ pelo CCL - $\triangle \mathrm{CCL}$, formula 35. Percentualmente, a variação será igual à divisão entre $\Delta \mathrm{CCL}$ e CCL $-\Delta \% \mathrm{CCL}$, formula 36. 
$\Delta C C L=C C L_{A}-C C L=L_{E S T}=E S T \times M L_{E S T}=E S T \times \frac{L A D J I R}{C P V}$

$\Delta \% C C L=\frac{L_{E S T}}{C C L} \times 100=\frac{E S T \times M L_{E S T}}{C C L} \times 100=\frac{E S T \times \frac{L A D J I R}{C P V}}{A C-P C} \times 100=\frac{E S T \times L A D J I R}{C P V \times(A C-P C)} \times 100$

Da fórmula 36, tem-se a seguinte conclusão: $\triangle \%$ CCL tem cálculo baseado no produto entre a $\mathrm{ML}_{\mathrm{EST}}$ e a representatividade do EST ante o CCL, fórmula 37.

$\triangle \% C C L=M L_{E S T} \times \frac{E S T}{C C L} \times 100$

\section{Liquidez Geral Adaptada}

A LG adaptada, $\mathrm{LG}_{\mathrm{A}}$, tem a lucratividade sobre o EST incluída na soma entre AC e RLP, para se identificar à capacidade de cobertura dos ativos realizáveis circulantes e não-circulantes em face s necessidades de liquidações do passivo exigível.

As fórmulas de 38 a 41 demonstram as expressões matemáticas básicas da $\mathrm{LG}_{\mathrm{A}}$, onde os ativos juntamente com a lucratividade do EST são comparados com a soma entre PC e ELP.

$$
\begin{aligned}
L G_{A}= & \frac{A C+R L P+E S T \times M L_{E S T}}{P C+E L P} \\
L G_{A}= & \frac{A C+R L P+E S T \times \frac{L A D J I R}{C P V}}{P C+E L P} \\
L G_{A}= & \frac{C X+Q C+D R+R L P+E S T \times\left(1+M L_{E S T}\right)+O R C}{E F C+F O R+P R O+O L C+E L P} \\
L G_{A}= & \frac{C X+Q C+D R+R L P+E S T \times\left(1+\frac{L A D J I R}{C P V}\right)+O R C}{E F C+F O R+P R O+O L C+E L P}
\end{aligned}
$$

Nas fórmulas 42 e 43, tem-se a descrição da $L_{\mathrm{A}}$ sob a perspectiva das fontes de financiamento. A formulação $E L P+P L-C C L-A P$ refere-se ao saldo do RLP. A lucratividade do saldo do EST recebe tratamento similar ao despendido para as fontes de recursos.

$$
L G_{A}=\frac{P C+C C L+E L P+P L-C C L-A P+E S T \times M L_{E S T}}{P C+E L P}
$$


$L G_{A}=\frac{P C+C C L+E L P+P L-C C L-A P+E S T \times \frac{L A D J I R}{C P V}}{P C+E L P}$

Quanto maior a $\mathrm{LG}_{\mathrm{A}}$, melhor configurar-se-á o conceito de solvência geral. Quanto menor $\mathrm{LG}_{\mathrm{A}}$, pior configurar-se-á o conceito de solvência global.

Para identificar o valor incremental absorvido pela $L G$ no cálculo da $L G_{A}$, basta dividir a $\mathrm{L}_{\mathrm{EST}}$ pelo passivo exigível - $\Delta \mathrm{LG}$, fórmula 44.

$\Delta L G=\frac{L_{E S T}}{P C+E L P}=\frac{E S T \times M L_{E S T}}{P C+E L P}=\frac{E S T \times \frac{L A D J I R}{C P V}}{P C+E L P}=\frac{E S T \times L A D J I R}{C P V \times(P C+E L P)}$

Percentualmente, a modificação sobre a $L G$ para se atingir a $\mathrm{LG}_{\mathrm{A}}$ tem cálculo a partir da divisão da $\mathrm{L}_{\mathrm{EST}}$ pela soma entre RLP e AC, fórmula 45.

$\Delta \% L G=\frac{L_{E S T}}{R L P+A C} \times 100=\frac{E S T \times M L_{E S T}}{R L P+A C} \times 100=\frac{E S T \times \frac{L A D J I R}{C P V}}{R L P+A C} \times 100=\frac{E S T \times L A D J I R}{C P V \times(R L P+A C)} \times 100$

No desenvolvimento final da fórmula 45, observa-se o produto entre a MB e significância do EST, em face da soma de RLP e AC, fórmula 46.

$\Delta \% L G=M L_{E S T} \times \frac{E S T}{R L P+A C} \times 100$

\section{Liquidez Corrente Adaptada}

A liquidez corrente adaptada à lucratividade sobre as vendas, $\mathrm{LC}_{\mathrm{A}}$, tem no numerador a soma entre o AC e a rentabilidade do EST, para estimar a superioridade dos direitos realizáveis e disponibilidades sobre o PC, fórmulas 47 e 48.

$$
\begin{aligned}
L C_{A} & =\frac{A C+E S T \times M L_{E S T}}{P C} \\
L C_{A} & =\frac{A C+E S T \times \frac{L A D J I R}{C P V}}{P C}
\end{aligned}
$$

Na fórmula 49, o AC tem sua composição detalhada, evidenciando uma alternativa distinta da praticada nas fórmulas 47 e 48 para se inserir a lucratividade 
do EST na apreciação da solvência. Se nas fórmulas 47 e 48 a participação da rentabilidade se dá por meio da correção básica (EST vezes $\mathrm{ML}_{\mathrm{EST}}$ ), exprimindo apenas os rendimentos, na 49 o EST $_{C}$ participa integralmente, esboçando o valor total, vinculado às transações comerciais.

$L C_{A}=\frac{C X+Q C+D R+E S T \times\left(1+M L_{E S T}\right)+O R C}{E F C+F O R+P R O+O L C}=\frac{C X+Q C+D R+E S T \times\left(1+\frac{L A D J I R}{C P V}\right)+O R C}{E F C+F O R+P R O+O L C}$

Processando somente as fontes de recursos pertencentes à estrutura de financiamento do $\mathrm{AC}$ para mensurar a $\mathrm{LC}_{\mathrm{A}}$, no numerador encontrar-se-ão somados o PC, o CCL e a lucratividade do EST; no denominador, o PC será referência na consumação de ponderações atinentes à solvibilidade.

$L C_{A}=\frac{P C+C C L+E S T \times M L_{E S T}}{P C}=\frac{P C+C C L+E S T \times \frac{L A D J I R}{C P V}}{P C}$

Por ser tratada também como fonte interna de financiamento das atividades correntes, a lucratividade associada ao EST será somada com o CCL para reforçar a superioridade sobre as exigências de curto prazo. Observando a fórmula 51, as discussões inclinam-se à adoção do seguinte conjunto de premissas: se a soma entre CCL e lucratividade do EST for inferior ao PC, a LC $_{A}$ será inferior a 1; se a soma entre CCL e a lucratividade do EST for igual ao PC, a $\mathrm{LC}_{\mathrm{A}}$ será igual a 1; se a soma entre CCL e a lucratividade do EST for superior ao $\mathrm{PC}$, a $\mathrm{LC}_{\mathrm{A}}$ será superior a 1 .

$L C_{A}=1+\frac{C C L+E S T \times M L_{E S T}}{P C}=1+\frac{C C L+E S T \times \frac{L A D J I R}{C P V}}{P C}$

$\mathrm{A} \mathrm{LC}_{\mathrm{A}}$ será sempre superior à LC. Quanto maior a $\mathrm{LC}_{\mathrm{A}}$, melhor será o conceito de solvência.

A diferença entre a $\mathrm{LC}_{\mathrm{A}}$ e a $\mathrm{LC}$ pode ser evidenciada pela fórmula $52(\Delta \mathrm{LC})$, onde se observa a divisão entre a $L_{E S T}$ e o PC.

$\Delta L C=\frac{L_{E S T}}{P C}=\frac{E S T \times M L_{E S T}}{P C}=\frac{E S T \times \frac{L A D J I R}{C P V}}{P C}=\frac{E S T \times L A D J I R}{C P V \times P C}$

Percentualmente, a alteração imputada à LC, com o cálculo da $\mathrm{LC}_{\mathrm{A}}$, pode ser encontrada a partir da divisão da $\mathrm{L}_{\mathrm{EST}}$ pelo $\mathrm{AC}-\Delta \% \mathrm{LC}$, formula 53. 
$\Delta \% L C=\frac{L_{E S T}}{A C} \times 100=\frac{E S T \times M L_{E S T}}{A C} \times 100=\frac{E S T \times \frac{L A D J I R}{C P V}}{A C} \times 100=\frac{E S T \times L A D J I R}{C P V \times A C} \times 100$

O desenvolvimento final da fórmula 53 sugere o cálculo da $\Delta \% \mathrm{LC}$ por meio do produto entre a $\mathrm{ML}_{\mathrm{EST}}$ e a representatividade do EST ante o AC, fórmula 54.

$\Delta \% L C=M L_{E S T} \times \frac{E S T}{A C} \times 100$

\section{Liquidez de Kanitz Adaptada}

Apesar da proposição centrada na dinâmica, a LK não consegue amoldar logicamente a análise de liquidez com fins na solvência em condições de prosperidade empresarial. O artifício de cálculo atrelado à exclusão de uma parcela não negociável do EST perfaz apenas uma parte das modificações necessárias à constituição definitiva da dinâmica. Destarte, ao trabalhar com a lucratividade do EST, talvez não se tenha alcançado definitivamente a dinâmica na análise de liquidez; todavia tem-se a construção de um passo significativo à aproximação da realidade.

A fórmula 55 utiliza a $\mathrm{L}_{\mathrm{EST}}$ como meio na cobertura do PC. O cálculo $E S T \times\left[1+F_{V} \times\left(M L_{E S T}-1\right)\right]$ representa uma evolução matemática, oriunda da junção das duas correções: a original, proposta pela LK; e a atual, sugerindo a inclusão da rentabilidade na apuração da solvência, com o índice de liquidez de Kanitz adaptada $\left(\mathrm{LK}_{\mathrm{A}}\right)$.

$L K_{A}=A C+E S T \times\left[1+F_{V} \times\left(M L_{E S T}-1\right)\right]=A C+E S T \times\left[1+F_{V} \times\left(\frac{L A D J I R}{C P V}-1\right)\right]$

Na fórmula 56, a adaptação dinâmica dá-se com a inserção do $\mathrm{EST}_{\mathrm{C}}$, sendo a base de correção original, promovida por $\mathrm{F}_{\mathrm{V}}$.

$$
\begin{aligned}
& L K_{A}=\frac{C X+Q C+D R+E S T \times F_{V} \times\left(1+M L_{E S T}\right)+O R C}{E F C+F O R+P R O+O L C} \\
& L K_{A}=\frac{C X+Q C+D R+E S T \times F_{V} \times\left(1+\frac{L A D J I R}{C P V}\right)+O R C}{E F C+F O R+P R O+O L C}
\end{aligned}
$$

As fórmulas 58 e 59 demonstram o raciocínio básico de sustentação da solvência de curto prazo com base na $\mathrm{LK}_{\mathrm{A}}$ sob a perspectiva da estrutura de financiamento do AC. 


$$
\begin{aligned}
& L K_{A}= \frac{P C+C C L+E S T \times\left[1+F_{V} \times\left(M L_{E S T}-1\right)\right]}{P C} \\
& L K_{A}= P C+C C L+E S T \times\left[1+F_{V} \times\left(\frac{L A D J I R}{C P V}-1\right)\right] \\
& P C
\end{aligned}
$$

Observando as fórmulas 60 e 61, vê-se a $\mathrm{L}_{\mathrm{EST}}$ unindo-se ao CCL para determinar as conclusões finais atinentes à capacidade de cobertura dos compromissos correntes assumidos. A diferença, em face da fórmula da LK, está na soma da $\mathrm{L}_{\mathrm{EST},}$ ligada ao saldo vendido com o CCL.

$$
\begin{aligned}
& L K_{A}=1+\frac{C C L+E S T \times\left[1+F_{V} \times\left(M L_{E S T}-1\right)\right]}{P C} \\
& L K_{A}=1+\frac{C C L+E S T \times\left[1+F_{V} \times\left(\frac{L A D J I R}{C P V}-1\right)\right]}{P C}
\end{aligned}
$$

Quanto maior a $\mathrm{LK}_{\mathrm{A}}$, melhor será a configuração da solvência. Ainda, em relação a $L K$, a $L K_{A}$ será sempre maior.

Com a inserção da lucratividade intrínseca ao EST no cálculo da LK, tem-se uma variação positiva absorvida pela $\mathrm{LK}_{\mathrm{A}}$. Para encontrar o valor absoluto da alteração, a fórmula 62 sugere a divisão do produto entre $L_{E S T}$ e a $F_{V}$ pelo PC $-\Delta \mathrm{LK}$.

$\Delta L K=\frac{L_{E S T} \times F_{V}}{P C}=\frac{E S T \times M L_{E S T} \times F_{V}}{P C}=\frac{E S T \times \frac{L A D J I R}{C P V} \times F_{V}}{P C}=\frac{E S T \times L A D J I R \times F_{V}}{C P V \times P C}$

A variação relativa imputada à $L K$, com o processamento da $L K_{A}$, tem expressão com divisão do produto entre $\mathrm{L}_{\mathrm{EST}}$ e $\mathrm{F}_{\mathrm{V}}$ pela diferença entre $\mathrm{AC}$ e a parcela não negociada do EST - $\Delta \%$ LK, fórmula 63.

$\Delta \% L K=\frac{L_{E S T} \times F_{V}}{A C-E S T \times\left(1-F_{V}\right)} \times 100=\frac{E S T \times \frac{L A D J I R}{C P V} \times F_{V}}{A C-E S T \times\left(1-F_{V}\right)} \times 100=\frac{E S T \times L A D J I R \times F_{V}}{C P V \times\left[A C-E S T \times\left(1-F_{V}\right)\right]} \times 100$

Da fórmula 63 extrai-se a seguinte conclusão: a $\Delta \% \mathrm{LK}$ tem processamento baseado no produto entre a $\mathrm{ML}_{\mathrm{EST}}$ e representatividade da porção vendida do EST - EST vezes FV, em face do volume do AC líquido da parcela não negociada do EST, fórmula 64. 
$\Delta \% L K=M L_{E S T} \times \frac{E S T \times F_{V}}{A C-E S T \times\left(1-F_{V}\right)} \times 100$

\section{Apreciação Empírica Comparativa}

Visando observar na prática as diferenças entre as modalidades de cálculo com e sem lucratividade associada à análise de liquidez, com base no esclarecimento do potencial de solvibilidade, a Tabela 1 contém as cifras das rubricas pertencentes ao balanço patrimonial e a demonstração do resultado do exercício, envoltas com os procedimentos de cálculo.

\section{Tabela 1: Dados Contábeis Relevantes às Análises de Liquidez Convencional e Adaptada à Lucratividade}

\begin{tabular}{|c|c|c|c|c|c|}
\hline \multicolumn{6}{|c|}{ Rubricas Contábeis Importantes } \\
\hline \multicolumn{4}{|c|}{ Balanço Patrimonial } & \multicolumn{2}{|c|}{ Demonstração do Resultado } \\
\hline \multirow{4}{*}{ AC } & $\mathbf{C X}$ & \multirow{4}{*}{5.500} & 530 & \multirow{5}{*}{ ROL } & \multirow{5}{*}{35.000} \\
\hline & QC & & 250 & & \\
\hline & DR & & 2.500 & & \\
\hline & EST & & 2.220 & & \\
\hline RLP & \multicolumn{3}{|c|}{3.200} & & \\
\hline \multirow{3}{*}{ PC } & EFC & & 1.250 & \multirow{4}{*}{ LADJIR } & \multirow{4}{*}{26.250} \\
\hline & FOR & 3.850 & 1.600 & & \\
\hline & PRO & & 1.000 & & \\
\hline ELP & \multicolumn{3}{|c|}{2.350} & & \\
\hline
\end{tabular}

Fonte: formulação própria.

Na Tabela 2, encontram-se dispostos os indicadores apurados com base nos dados da Tabela 1.

Tabela 2: Resultados das Análises de Liquidez Convencional e Adaptada à Lucratividade

\begin{tabular}{|c|c|c|c|}
\hline \multicolumn{4}{|c|}{ Análise de Liquidez } \\
\hline \multicolumn{2}{|c|}{ Convencional } & \multicolumn{2}{|c|}{ Adaptada à Lucratividade } \\
\hline CCL & 1.650 & ML $_{\text {EST }}$ & $\mathbf{0 , 7 5}$ \\
\hline LG & 1,40 & $\mathbf{E S T}_{\mathbf{C}}$ & 3.885 \\
\hline $\mathbf{L C}$ & 1,43 & $\mathrm{CCL}_{\mathrm{A}}$ & 3.315 \\
\hline LS & 0,85 & $\mathbf{L G}_{\mathbf{A}}$ & 1,67 \\
\hline $\operatorname{LK}\left(F_{V}=40 \%\right)$ & 1,08 & $\mathbf{L C}_{\mathrm{A}}$ & 1,86 \\
\hline LA & $\mathbf{0 , 2 0}$ & $\mathrm{LK}_{\mathrm{A}}\left(\mathrm{F}_{\mathrm{V}}=40 \%\right)$ & 1,26 \\
\hline
\end{tabular}

Fonte: formulação própria.

A Tabela 3 mostra a superioridade dos medidores de liquidez adaptados à lucratividade em face dos medidores convencionais.

A lucratividade do EST deixará de ser implícita com a correção dele por meio 
da $\mathrm{ML}_{\mathrm{EST}}$; logo, o $\mathrm{EST}_{\mathrm{C}}$ garante a inserção de um valor incremental ao $\mathrm{AC}$, sendo justamente a $\mathrm{L}_{\mathrm{EST}}$, substanciando os indicadores adaptados.

\section{Tabela 3: Diferenças nos Resultados das Análises de Liquidez Convencional e Adaptada à Lucratividade}

\begin{tabular}{c|c|c}
\hline \multicolumn{2}{|c}{ Variações Importantes } \\
\hline Convencional & Adaptada & $\Delta \%$ \\
\hline EST & EST $_{\mathbf{C}}$ & $+\mathbf{7 5 \%}$ \\
\hline CCL & CCL $_{\mathbf{A}}$ & $+\mathbf{1 0 0 , 9 1 \%}$ \\
\hline LG & $\mathbf{L G}_{\mathbf{A}}$ & $+\mathbf{1 9 , 2 9}$ \\
\hline LC & $\mathbf{L C}_{\mathbf{A}}$ & $+\mathbf{3 0 , 0 7}$ \\
\hline LK $\left(\mathbf{F}_{\mathbf{V}}=40 \%\right)$ & $\mathbf{L K}_{\mathbf{A}}\left(\mathbf{F}_{\mathbf{V}}=\mathbf{4 0 \%}\right)$ & $+\mathbf{1 6 , 6 7}$ \\
\hline
\end{tabular}

Fonte: formulação própria.

No exemplo em estudo, a maior variação ocorreu com o valor do CCL. Com a lucratividade do EST, o CCL $\mathrm{A}_{\mathrm{A}}$ mostrou-se 100,91\% maior. A $\mathrm{LG}_{\mathrm{A}}$ teve acréscimo de

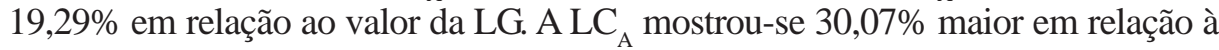
LC. A LK ${ }_{A}$ apresenta uma superioridade de 16,67\%, face o valor expresso pela LK.

Finalmente, todos os indicadores inseridos na adaptação proposta pela lógica dinâmica apresentaram valores superiores, quando comparados com os estáticos, ou sem a consideração da lucratividade sobre vendas.

\section{Considerações Finais}

A solvência pode ser estimada em duas circunstâncias estruturais mutuamente excludentes: falência ou colapso (estática) e prosperidade (dinâmica) empresarial (Brasil; Brasil, 1997).

Galgando equacionar a deficiência da análise liquidez destinada à estimação da solvência corrente sob a configuração dinâmica, a análise de lucratividade sobre vendas fora envolvida no desenvolvimento da solução.

Finalmente, o capital circulante líquido, a liquidez geral, a liquidez corrente e a liquidez de Kanitz são matematicamente modificados, a partir da correção dos estoques com a margem de lucratividade inerente a eles e neles implícita. Ao inserir a lucratividade no cálculo dos tradicionais medidores de liquidez, tem-se a consecução da adaptação exigida pela análise sob a lógica dinâmica, proferindo valores mais confiáveis quanto à mensuração da solvência de curto prazo, em face da perspectiva dos volumes dos fluxos ativos e passivos.

Artigo recebido em 29.08.2003. Aprovado em 24.01.2004. 
REFERÊNCIAS

ASSAF N. A.

Estrutura e análise de balanços. 5.ed. São Paulo: Atlas, 2000.

BRASIL, H. V.;

BRASIL, H. G.

Gestão financeira das empresas: um modelo dinâmico. 3.ed. Rio de Janeiro: Qualitymark, 1997.

\section{BRIGHAM, E.; \\ HOUSTON, J.}

Fundamentos da moderna administração financeira. Rio de Janeiro: Campus, 1999.

FERNÁNDEZ, P.

Valoración de empresas. Barcelona: Gestión 2000, 1999.

FLEURIET, M.;

KEHDY,R.;

BLANC, G

O modelo fleuriet: a dinâmica financeira das empresas brasileiras. Rio de Janeiro: Campus, 2003.

GITMAN, L. J.

Princípios de administração financeira. 7.ed. São Paulo: Harbra, 1997.

\section{LOPES DE SÁ, A.}

Estudo analítico da rentabilidade das empresas. Rio de Janeiro: Apec, 1971.

MARTINS, E.;

A. N. A.

Administração financeira. São Paulo: Atlas, 1985.
MATARAZZO, D.

Análise financeira de balanços. 5.ed. São Paulo: Atlas, 1998.

MYERS, J.N.

Análise das demonstrações financeiras. São Paulo: Atlas, 1974.

SILVA, A. M. L.

O Estudo da liquidez estática e o valor do dinheiro no tempo. In: ENCONTRO IBERO-AMERICANO DE FINANÇAS E SISTEMAS DE INFORMAÇÃO, 3., 2002, Setúbal. em CD Rom.

Uma aproximação à solvência: análises de liquidez segundo a equivalência de capitais. 318 f. Dissertação (Mestrado em Administração de Empresas - Finanças) - PPGA, UFPB, João Pessoa. 2003.

SILVA, A. M. L.;

ALVES, M.A.S.

Contribuição ao estudo dos medidores de desempenho econômico: identificando variações através dos hiatos de resultado. In: ENCONTRO IBERO-AMERICANO DE FINANÇAS E SISTEMAS DE INFORMAÇÃO, 3., 2002, Setúbal. em cd rom.

SILVA, J.P.

Análise financeira das empresas. 4.ed. São Paulo: Atlas, 1999.

VAN HORNE, J.C.

Política e administração financeira. v.2. São Paulo: Livros Técnicos e Científicos Editora, 1979. 
WALTER, M. A.;

BRAGA, H. R.

Demonstrações financeiras: um enfoque gerencial. São Paulo: Saraiva, 1979.
WESTON, J. F;

BRIGHAM, E. F.

Fundamentos da administração

financeira. 10.ed. São Paulo: Makron

Books, 2000.p. 1030 